Electron beam induced compositional and structural changes in $\mathrm{Tl}_{2} \mathrm{Ba}_{2} \mathrm{CuO}_{6+\sigma}$

This content has been downloaded from IOPscience. Please scroll down to see the full text. 1996 Supercond. Sci. Technol. 9766

(http://iopscience.iop.org/0953-2048/9/9/010)

View the table of contents for this issue, or go to the journal homepage for more

Download details:

IP Address: 147.96.14.16

This content was downloaded on 14/10/2013 at 19:47

Please note that terms and conditions apply. 


\title{
Electron beam induced compositional and structural changes in $\mathrm{Tl}_{2} \mathrm{Ba}_{2} \mathrm{CuO}_{6+\delta}$
}

\author{
C Díaz-Guerra $\nmid$, J Piqueras $\dagger$, Yu Ya Tomashpolsky $\ddagger$, \\ N V Sadovskaya $\ddagger$ and C Opagiste $\S$ \\ † Departamento de Física de Materiales, Facultad de Ciencias Físicas, Universidad \\ Complutense, E-28040 Madrid, Spain \\ ¥ Karpov Institute of Physical Chemistry, Vorontsovo Pole 10, 103064 Moscow, \\ Russia \\ $\S$ Laboratoire Structure de la Matière, Université de Savoie, BP 240, 9 Rue de \\ L'Arc-en-Ciel, F-74942 Annecy le Vieux Cédex, France
}

Received 9 May 1996

\begin{abstract}
Tl}_{2} \mathrm{Ba}_{2} \mathrm{CuO}_{6+\delta}$ ceramics have been irradiated in a scanning electron microscope. The resultant changes in the cationic stoichiometry and oxygen content of the samples were investigated by cathodoluminescence $(C L)$, secondary electron emission (SEE) and EDX microanalysis. TI depletion and Ba enrichment are observed in the irradiated areas. CL spectroscopy shows that irradiation causes the increase of a $540 \mathrm{~nm}$ emission band attributed to complex centres involving oxygen vacancies. Other features of the $C L$ spectra strongly suggest the presence of radiation induced new phases. The SEE yield, $\Delta$, has been found to depend on the oxygen content of the samples. This dependence can be explained on the basis of two charge carrier subsystems and their mutual interaction. Measurements of $\Delta$ allow us to detect an oxygen deficient region, sometimes extending up to a distance of about $1000 \mu \mathrm{m}$, that surrounds the irradiated material.
\end{abstract}

\section{Introduction}

The effect of laser or electron irradiation on the properties of high-temperature superconductors (HTSCs) is a subject of technological interest related to the development of lithographic and patterning processes of potential application in superconducting electronics [1]. In addition, the study of radiation effects has application in the growth of superconducting thin films from target substrates. Most of the previous reports on this subject refer to the $\mathrm{Y}-\mathrm{Ba}-\mathrm{Cu}-\mathrm{O}(\mathrm{YBCO})$ and $\mathrm{Bi}-\mathrm{Sr}-\mathrm{Ca}-\mathrm{Cu}-\mathrm{O}$ (BSCCO) systems. Auger techniques [2,3] have been used to study compositional changes induced in YBCO and BSCCO by laser or electron irradiation. In high-resolution patterning studies of YBCO films by focused laser beams $[4,5]$, it has been found that this material can be locally modified by varying its oxygen content. Laser writing induces semiconducting patterns on the superconducting films by a reduction of oxygen content due to local heating. Under certain laser irradiation conditions, changes in the superconducting properties of YBCO are detected which are interpreted on the basis of photoassisted oxygen rearrangement $[6,7]$.

Radiation induced structural and compositional changes in YBCO and BSCCO have been also investigated by micro-Raman spectroscopy and cathodoluminescence
(CL) in the scanning electron microscope (SEM) [8-10]. CL microscopy enables us to detect the appearance of radiation induced insulating phases as well as structural changes influencing the intrinsic luminescence of the HTSC samples. Luminescence effects related to electronic processes in the oxygen sublattice in different HTSC single crystals and ceramics have been often reported in the past years (see e.g. [8-14]).

In the present work the effect of electron irradiation on $\mathrm{Tl}_{2} \mathrm{Ba}_{2} \mathrm{CuO}_{6+\delta}$ samples has been investigated in the SEM by using CL microscopy, energy dispersive $\mathrm{x}$ ray microanalysis (EDX) and secondary-electron emission (SEE). SEE has been used in recent years in phase analysis on the grain scale, in the determination of oxygen content, and in the detection of phase transitions in YBCO and BSCCO ceramics and single crystals [15-18]. The exponential dependence of the SEE yield on the oxygen concentration explains the high sensitivity of this technique in the local determination of oxygen deficiency. Detection of phase transitions is also possible due to significant anomalies of the secondary-electron emission intensity near such transitions. For these reasons SEE can be a suitable complementary technique to $\mathrm{CL}$ in oxygen content and distribution problems. 

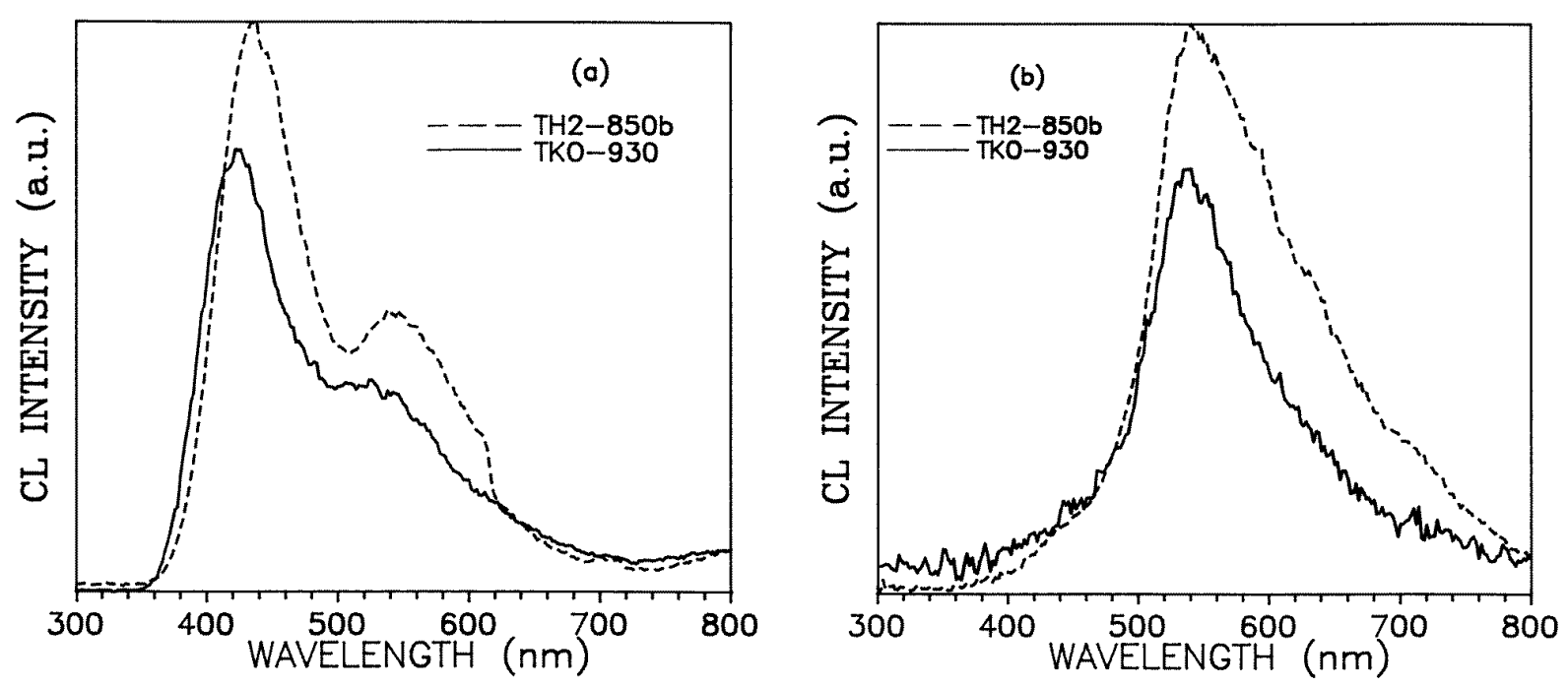

Figure 1. CL spectra of the TH2-850b and TKO-930 samples before irradiation: (a) $80 \mathrm{~K}$; (b) $300 \mathrm{~K}$.

Table 1. Relevant physical parameters of the samples investigated ( $f$ represents the Meissner fraction).

\begin{tabular}{llclllll}
\hline Sample & Batch & $T_{c}(\mathrm{~K})$ & $f(\%)$ & Symmetry & $a(\AA)$ & $b(\AA)$ & $c(\AA)$ \\
\hline TM-850 & B & 91.1 & 43.9 & $14 / m m m$ & 3.8717 & 3.8717 & 23.225 \\
TH2-850b & B & 52.4 & 49.1 & Ccc2 & 5.4582 & 5.4811 & 23.213 \\
TKO-930 & A & 0 & - & Ccc2 & 5.4467 & 5.4911 & 23.144 \\
\hline
\end{tabular}

\section{Experimental method}

The synthesis procedure of the samples has been described in detail in [19] and [20]. $\mathrm{Tl}_{2} \mathrm{Ba}_{2} \mathrm{CuO}_{5}$ and $\mathrm{CuO}$ powders were used as starting components. Two different batches were prepared from stoichiometric amounts of powders. For batch A the powder was first kept for 3 days at $750{ }^{\circ} \mathrm{C}$ under 1 bar oxygen whereas batch $\mathrm{B}$ was not pretreated. The powder mixture was pressed into pellets under $5 \mathrm{Kbar}$ and then annealed under 100 bar of argon or of oxygen with an appropiate thermal treatment. In the first case tetragonal superconducting (TS) samples are obtained while the treatment under oxygen yields orthorhombic non-superconducting (ONS) samples. A series of annealing treatments at low oxygen pressure has shown that starting samples with different symmetry. i.e. ONS and TS, transform into orthorhombic superconducting (OS), indicating that the final state of the specimen does not depend on the initial symmetry. In this work three samples whose codes and relevant data are listed in table 1 have been studied. One sample (TM-850) is TS, one (TKO-930) is ONS and the last (TH2-850b) is OS.

Samples from batch $\mathrm{A}$ are $\mathrm{x}$-ray pure. Some traces of $\mathrm{CuO}$ and $\mathrm{Ba}_{2} \mathrm{Cu}_{3} \mathrm{O}_{x}$ impurities are detected; both are estimated to be less than $0.1 \%$ vol. Optical micrographs show only Tl-2201 grains. The samples from batch B contain, besides the 2201 stoichiometric grains, less than $5 \%$ by volume of starting $\mathrm{Tl}_{2} \mathrm{Ba}_{2} \mathrm{O}_{5}$ impurity phase and very small $\mathrm{CuO}$ grains $(<0.1$ vol\%). Further details on these samples are described in [19] and [20].
Samples were polished with diamond paste and observed in the emissive and CL modes in a Hitachi S-2500 SEM at an accelerating voltage of $25 \mathrm{kV}$ and temperatures between 80 and $300 \mathrm{~K}$. For CL measurements an optical lens was used to concentrate the light on a photomultiplier attached to a window of the microscope. To record spectra an optical guide feeding the light to an Oriel 78215 computer controlled monochromator was used.

Large areas of some samples, of $80 \times 100 \mu \mathrm{m}^{2}$ in TKO930 and $50 \times 180 \mu \mathrm{m}^{2}$ in $\mathrm{TH} 2-850 \mathrm{~b}$, were irradiated under high-excitation conditions in the SEM. The irradiation was performed at room temperature with an accelerating voltage of $25 \mathrm{kV}$ and beam current higher than $10^{-7} \mathrm{~A}$. These areas were irradiated until a final saturation condition, as referred to morphological, total CL intensity and CL spectral changes, was reached.

In order to detect possible stoichiometric changes in the irradiated areas, EDX microanalyses were carried out in a JEOL JSM-35 CF SEM.

The SEE measurements were performed in a modified BS-350 scanning electron microscope working in UHV conditions $\left(10^{-7} \mathrm{~Pa}\right)$ and operating with a field emission $\mathrm{W}$ cathode. The SEE signal from the Everhart-Tornley type detector was registrated by a digital voltmeter and simultaneously recorded and treated by computer. The accelerating voltage of the primary electron beam was $16 \mathrm{kV}$ and the beam current was $10^{-10}-10^{-11} \mathrm{~A}$.

The SEE yield was measured from scanning areas of $23 \times 16,48 \times 34$ or $230 \times 160 \mu \mathrm{m}^{2}$ in size. These measurements were performed along several directions 

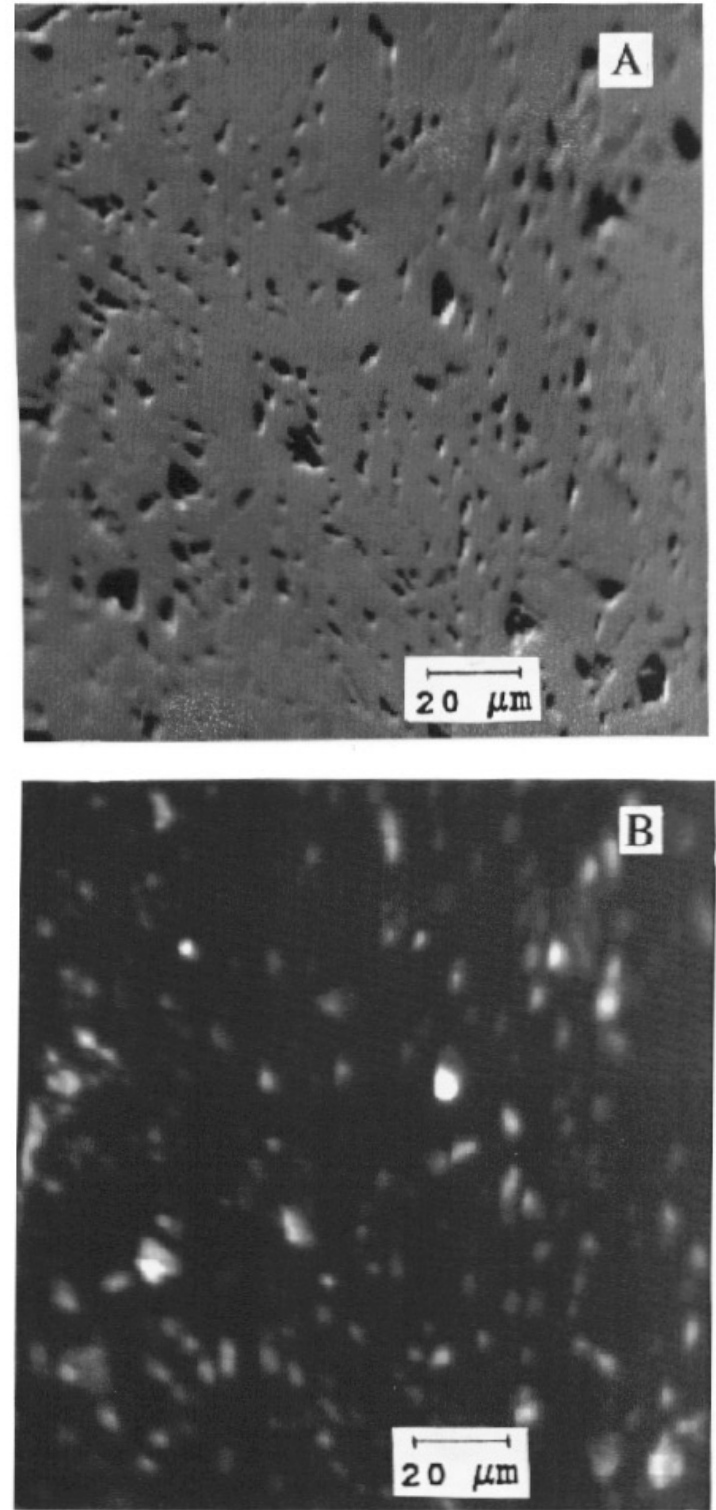

Figure 2. Emissive (a) and CL (b) mode micrographs of the untreated TKO-930 sample.

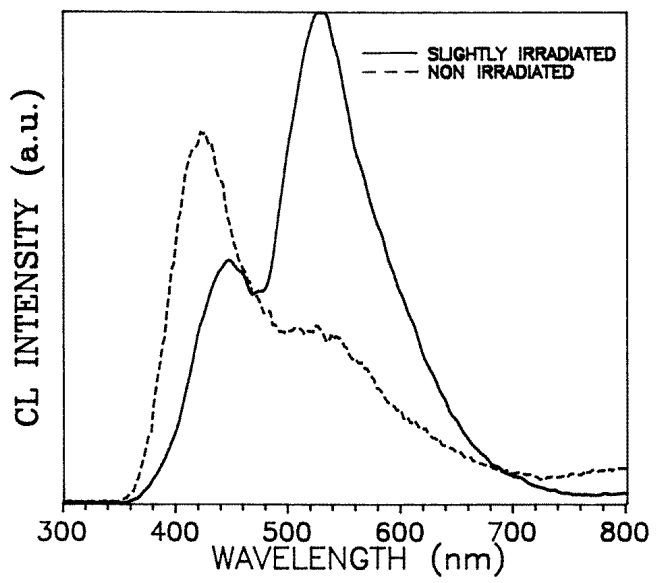

Figure 3. $\mathrm{CL}$ spectra recorded at $80 \mathrm{~K}$ from slightly irradiated and non-irradiated areas of the TKO-930 non-superconducting ceramic.
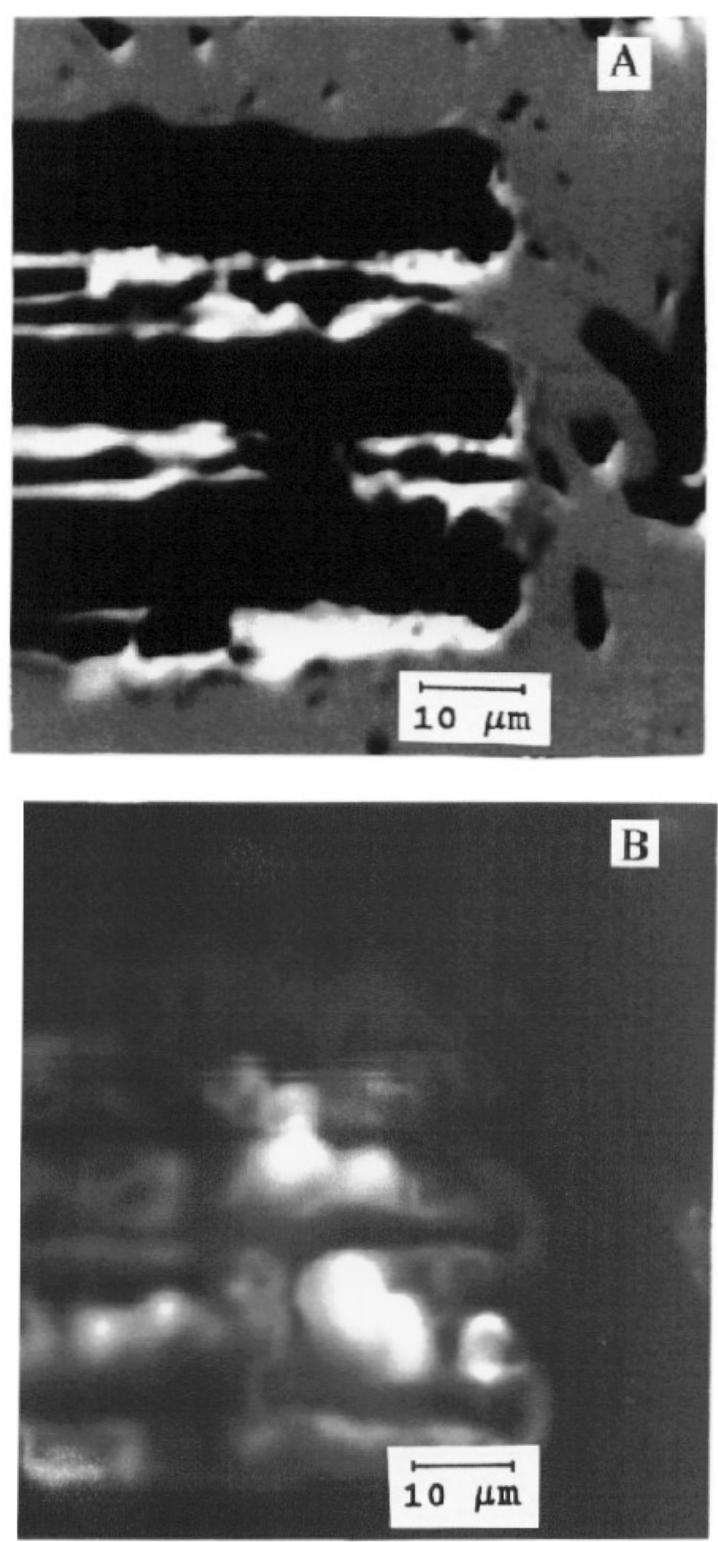

Figure 4. Secondary-electron (a) and $\mathrm{CL}(b)$ micrographs of an irradiated area of the TH2-850b sample.

across the irradiated areas in $16,23,34$ and $48 \mu \mathrm{m}$ step intervals.

\section{Results}

\subsection{Cathodoluminescence}

The CL properties before any treatment of the samples used in this work have been previously reported [21]. An emission band at $540 \mathrm{~nm}(\sim 2.3 \mathrm{eV})$ whose intensity increases on reducing oxygen content was proposed to be due to a complex centre involving oxygen vacancies. Another band centred at about $430 \mathrm{~nm}(\sim 2.9 \mathrm{eV})$ is also observed in the samples. It has been previously attributed to processes taking place in the oxygen sublattice, but has a different origin than the $540 \mathrm{~nm}$ emission. Figure 1 shows representative CL spectra of untreated 

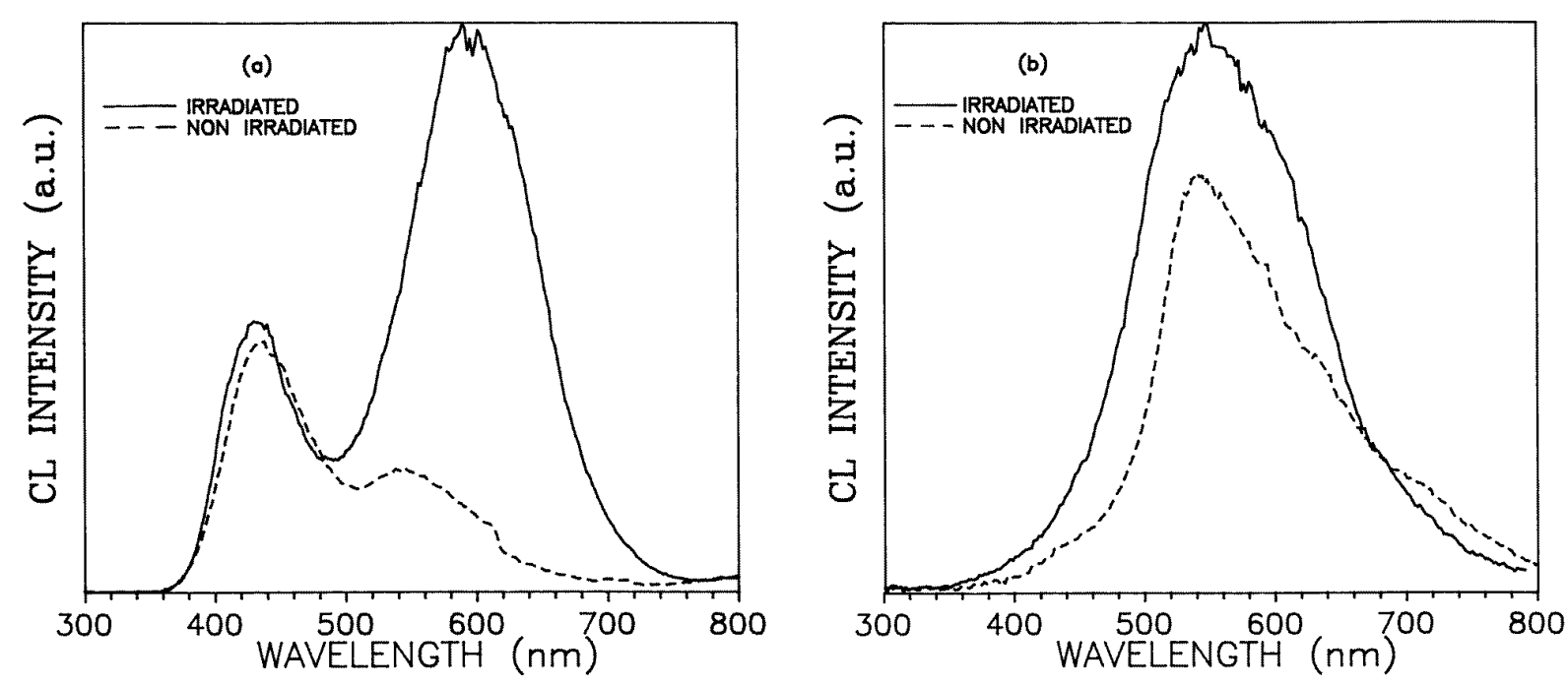

Figure 5. $\mathrm{CL}$ spectra recorded at $80 \mathrm{~K}(\mathrm{a})$ and $300 \mathrm{~K}(\mathrm{~b})$ of the TH2-850b superconducting sample before and after strong electron irradiation.
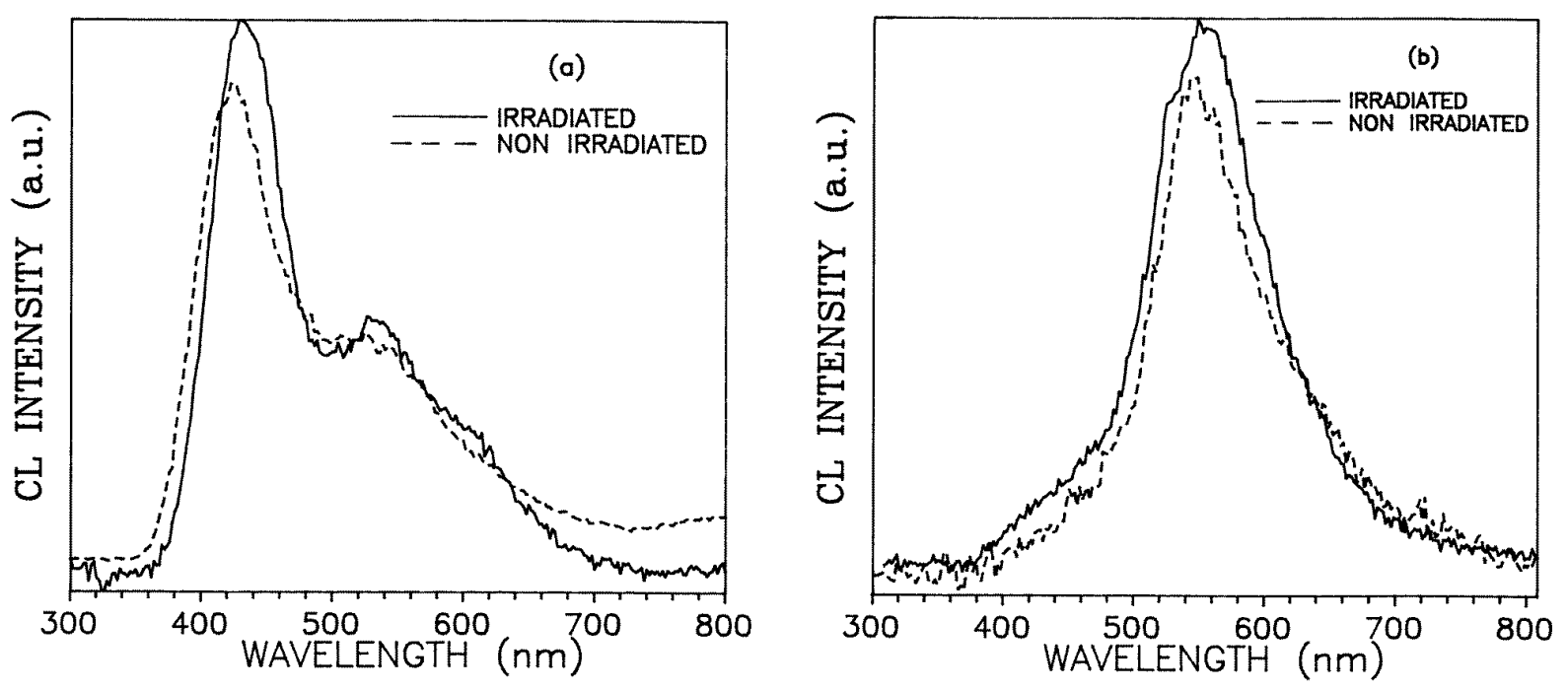

Figure 6. $\mathrm{CL}$ spectra recorded from the TKO-930 ceramic at $80 \mathrm{~K}(a)$ and $300 \mathrm{~K}(b)$ before and after strong irradiation.

TH2-850b and TKO-930 samples at 80 and $300 \mathrm{~K}$. Both bands are present at low temperatures while at room temperature the $540 \mathrm{~nm}$ emission clearly dominates. The appearance of CL images of all samples is similar showing an inhomogeneous distribution of luminescence (figure 2). Areas of enhanced intensity are related to pores. Inside these pores well defined grain surfaces and no foreign particles are observed, which indicates that the enhanced $\mathrm{CL}$ is related to grain surfaces freshly exposed during the polishing treatment [21]. Under normal observation conditions in the SEM no changes are produced in the topography or luminescence of the samples. When they are deliberately irradiated, e.g. by using a beam current higher than $10^{-7} \mathrm{~A}$, surface changes accompanied by increase of CL emission are observed. Some intermediate excitation conditions can cause increase of CL intensity without apparent topographic changes. CL spectra show that this slight irradiation causes the increase of the $540 \mathrm{~nm}$ luminescence band (figure 3) while after heavy irradiation an additional band centred at about $590 \mathrm{~nm}(\sim 2.1 \mathrm{eV})$ is observed in some samples.

For the reasons described in section 4, mainly the results corresponding to heavy irradiation have been considered. Figure 4 shows the secondary-electron and CL images of an irradiated area of the TH2-850b sample $\left(T_{c}=\right.$ $52.4 \mathrm{~K})$. The enhanced CL intensity is inhomogeneously distributed inside the irradiated region. In figure $5 \mathrm{CL}$ spectra of the same sample before and after irradiation at different temperatures are compared. The low-temperature $(80 \mathrm{~K})$ spectra show that the main effect of irradiation is 

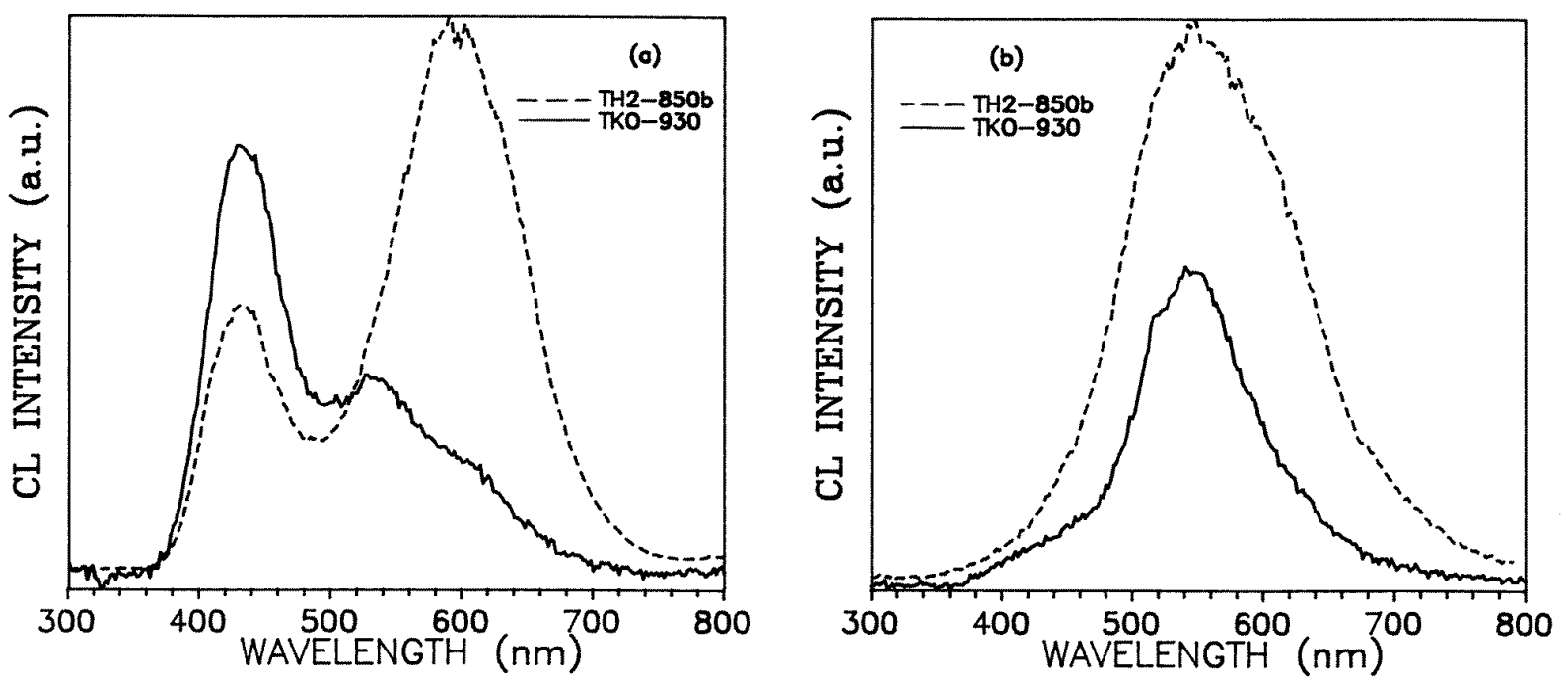

Figure 7. A comparison between $80 \mathrm{~K}(a)$ and room-temperature $(b) \mathrm{CL}$ spectra recorded from irradiated areas of the TH2-850b and TKO-930 samples.

the appearance of a broad band centred at $590 \mathrm{~nm}$. A component of this complex band is the original $540 \mathrm{~nm}$ band, resolved in unirradiated samples. This is apparent in the spectra recorded at $300 \mathrm{~K}$ (figure $5(b)$ ) which shows a band at $540 \mathrm{~nm}$ more intense in the irradiated regions.

The effect of irradiation on CL spectra of the nonsuperconducting sample (TKO-930) is shown in figure 6 . Spectral changes are small compared with those of the superconducting sample and the $590 \mathrm{~nm}$ peak is not observed. The difference in the CL emission of both irradiated samples is clearly observed in figure 7.

CL spectra recorded from the region surrounding the irradiated areas show a relative increase of the $540 \mathrm{~nm}$ emission band, as compared with the unaffected material (figure 8).

As described below, a high $\mathrm{Ba}$ content was measured by EDX in the irradiated areas. $\mathrm{BaCuO}_{2}$ has been usually found as a second phase in sintered HTSCs. In order to detect the possible presence of small amounts of this compound in these areas, the $\mathrm{CL}$ emission of a $\mathrm{BaCuO}_{2}$ ceramic was investigated. The spectrum recorded at $80 \mathrm{~K}$ shows a wide CL emission in the blue-green spectral range. A band peaked near $500 \mathrm{~nm}$ dominates the spectrum, while a shoulder centred at about $410 \mathrm{~nm}$ can also be observed (figure 9).

\subsection{X-ray microanalysis}

$\mathrm{X}$-ray microanalysis was performed in the irradiated area and at points at increasing distance from it. The accelerating voltage was $20 \mathrm{kV}$. The $\mathrm{L}$ lines of $\mathrm{Tl}$ and $\mathrm{Ba}$ and the $\mathrm{K}$ line of $\mathrm{Cu}$ were used. Measurements in different unaffected regions show that the average cationic composition is nearly stoichiometric $(2: 2: 1)$.

In superconducting and non-superconducting samples the irradiated areas have been found to be $\mathrm{Tl}$ poor and show a relative increase of $\mathrm{Ba}$ content while only slight changes

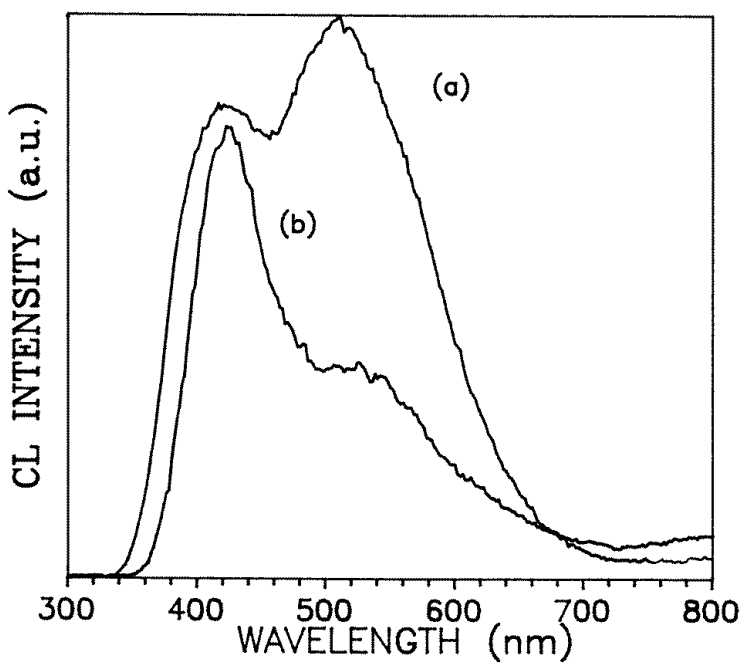

Figure 8. $\mathrm{CL}$ spectra recorded at $80 \mathrm{~K}$ from the area surrounding the irradiated region $(a)$ and the unaffected material $(b)$ in the TKO-930 sample.

in $\mathrm{Cu}$ composition are detected. Variations of cationic relative content extend to a distance of few micrometres from the irradiated area as figure 10 shows.

\subsection{Secondary electron emission}

Figure 11 shows a plot of the SEE yield of the untreated samples as a function of their $T_{c}$, normalized to the yield of the non-superconducting sample. Since only three samples are used in this work, the shape of the curve is unknown and the line drawn might not correspond to the real situation. However, the reproducibility of results demonstrates the general trend, shown in the figure, of an increase of the SEE yield followed by a decrease at high $T_{c}$. Since a higher $T_{c}$ 


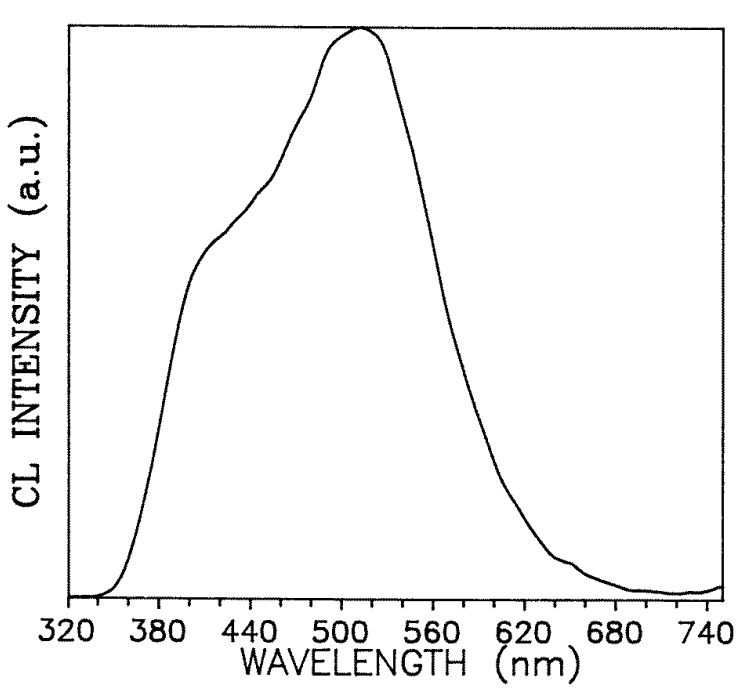

Figure 9. The $\mathrm{CL}$ low-temperature $(80 \mathrm{~K})$ spectrum of $\mathrm{BaCuO}_{2}$.

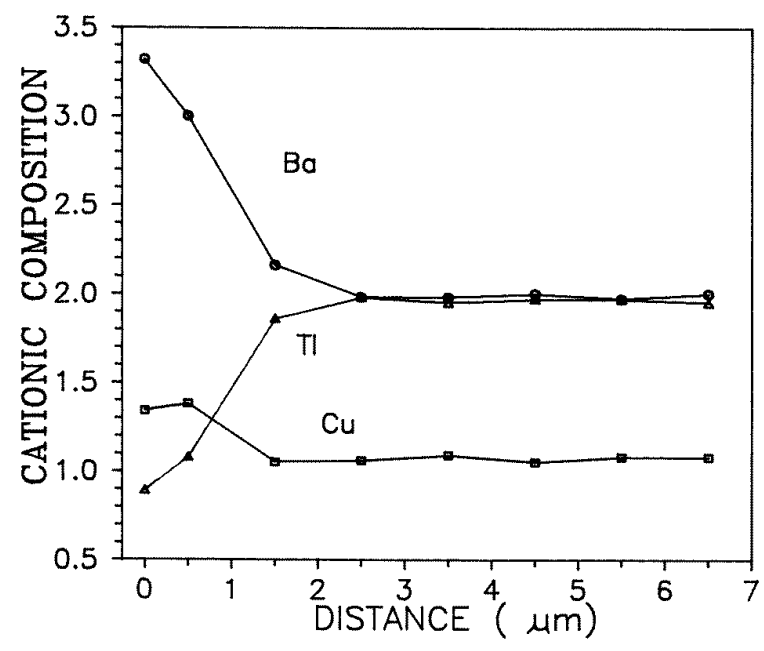

Figure 10. Cationic composition as a function of distance to the border of an irradiated area of the $\mathrm{TH} 2-850 \mathrm{~b}$ superconducting sample.

value corresponds to a lower oxygen content [19-22], this result means that on decreasing oxygen content the SEE yield increases in a first stage and then decreases.

In the ceramics investigated a higher SEE yield in the irradiated zone has been observed. Since irradiated regions have strong inhomogeneous surfaces this effect is not directly measured in large areas including topographical features. However, when areas of about $10 \times 10 \mathrm{~mm}^{2}$ are investigated it is found that the SEE yield from irradiated regions is very high as compared with non-irradiated ones. The changes in SEE have a different spatial distribution than the changes in the cationic composition, and extend to a larger distance from the irradiated area. In the unaffected region SEE is stable and lower than in the irradiated area.

Figure 12 shows the SEE yield in the TKO-930 sample normalized to the value in the unaffected region, as a function of the distance to the edge of the irradiated

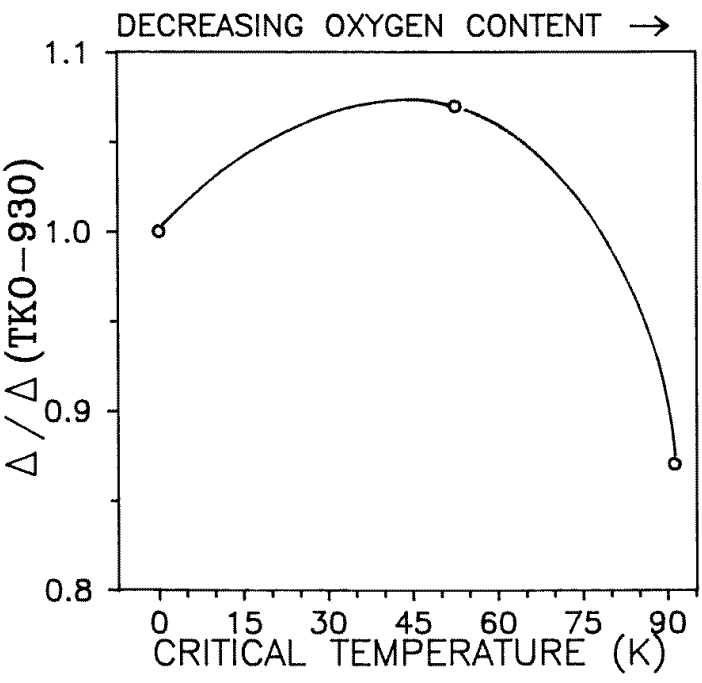

Figure 11. Dependence of the SEE yield on the critical temperature of the samples investigated. $\Delta$ values are normalized to the TKO-930 $\left(T_{c}=0 \mathrm{~K}\right)$ yield.

area. The yield increases from the edge and decreases after reaching a maximum value at a distance of about $600 \mu \mathrm{m}$. Comparison with figure 11 indicates that this behaviour is compatible with a continuous variation of oxygen content as a function of the distance to the irradiated area. The curve of SEE yield shows that changes in oxygen content extend up to a distance of about $1000 \mu \mathrm{m}$ from the edge. A qualitative evolution of oxygen composition is also represented in figure 12 . The two ends of the curve correspond to oxygen content in the borders of the irradiated area and in the unaffected region respectively. Figure 12 shows that cationic composition has no significative variations in this intermediate region beside the irradiated area.

Figure 13 shows the results of similar measurements in the $\mathrm{TH} 2-850 \mathrm{~b}$ sample. In this case $\Delta$ reaches the stable value at a distance of about $100 \mu \mathrm{m}$ from the irradiated zone. A qualitative curve of oxygen content as well as quantitative results of cationic composition are also represented in figure 13 .

\section{Discussion}

CL images of all untreated samples reveal the existence of luminescence emission with an inhomogeneous spatial distribution. This observation agrees with the previous results referred to in section 1 .

In a previous work [21] performed with the same set of samples used here, it was concluded that the CL intensity distribution is not due to impurity phases. This conclusion was obtained from characterization studies regarding kinds and amounts of impurity phases. A CL band at $540 \mathrm{~nm}$, whose intensity increases on reducing oxygen content of the samples, was proposed to be due to a complex centre involving oxygen vacancies. This band has been also reported in $\mathrm{YBa}_{2} \mathrm{Cu}_{3} \mathrm{O}_{7-x}$ and $\mathrm{Bi}_{2} \mathrm{Sr}_{2} \mathrm{CaCu}_{2} \mathrm{O}_{8+x}$ samples [8-10] and was related to processes in the oxygen 


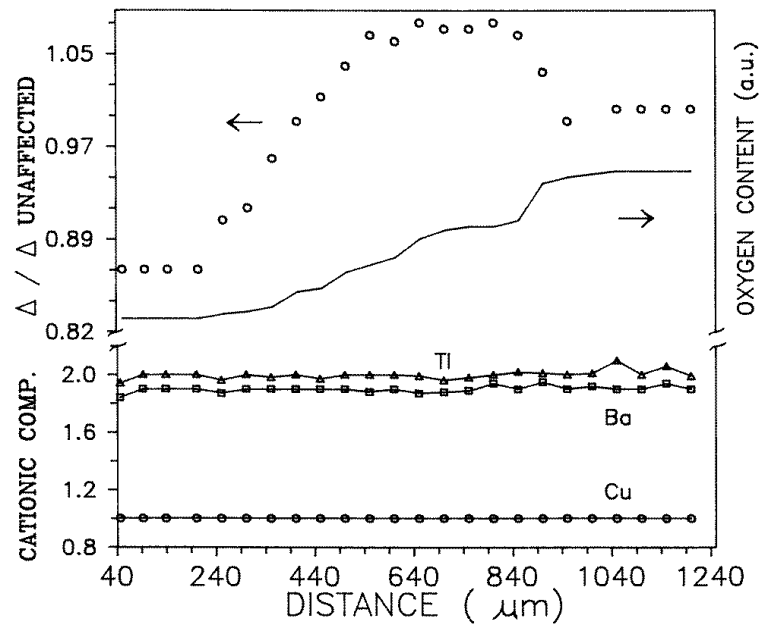

Figure 12. SEE yield in the TKO-930 sample normalized to the value in the unaffected region, cationic composition and oxygen content distribution, as functions of distance to the irradiated area. Cationic composition has been normalized to $\mathrm{Cu}$ content.

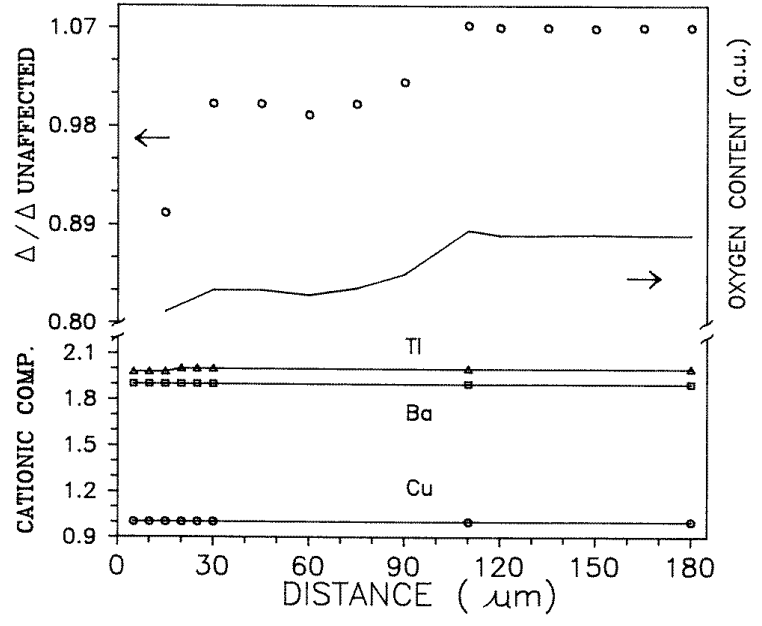

Figure 13. SEE yield in the TH2-850b superconducting sample normalized to the value in the unaffected region, cationic composition and oxygen content distribution, as functions of distance to the irradiated area. Cationic composition has been normalized to $\mathrm{Cu}$ content.

sublattice. In particular, a study of YBCO [9] has shown that in this system the initial CL spectra do not depend on the oxygen content, but during electron irradiation in the SEM an intense $540 \mathrm{~nm}$ band appears in oxygen deficient samples. Centres causing the $540 \mathrm{~nm}$ emission involve oxygen vacancies but are formed only after irradiation treatments, similar to the F centre formation in some ionic crystals. In the samples used in this work the $540 \mathrm{~nm}$ band is already present in the spectra of the untreated samples, and the possible spectral changes caused by electron irradiation would not be readily observable as in the case of $\mathrm{YBa}_{2} \mathrm{Cu}_{3} \mathrm{O}_{7-x}$. In order to relate the electron irradiation effects on the samples used in this work to their structural features, such as oxygen content, a high beam current -in the range of $10^{-7} \mathrm{~A}-$ was used. A large area was irradiated in two of the samples and the CL spectral changes were monitored until a final saturation condition was reached. The final state of the samples regarding their CL behaviour was then compared. In spectra of untreated samples a band centred at about $430 \mathrm{~nm}(\sim 2.9 \mathrm{eV})$ is also observed. A band at this energy has been previously reported in ceramic or single crystals of $\mathrm{YBa}_{2} \mathrm{Cu}_{3} \mathrm{O}_{7-x}$ (YBCO) and $\mathrm{Bi}_{2} \mathrm{Sr}_{2} \mathrm{CaCu}_{2} \mathrm{O}_{8-x}$ (BSCCO). It has been attributed in the case of $\mathrm{YBCO}$ and $\mathrm{BSCCO}$ to an oxygen quasimolecule [12] or to a complex involving an $\mathrm{F}$ centre and a negative molecular oxygen ion in YBCO [13].

Electron irradiation causes an enhancement of $\mathrm{CL}$ intensity as observed in CL spectra and images. After a moderate irradiation, i.e. irradiation not causing topographic changes, the same luminescence bands are present in the spectrum. However, a clear change in the relative weights of these bands is observed. Besides an increase of the total CL intensity, the $540 \mathrm{~nm}$ emission band is enhanced after irradiation and dominates the spectra.

In regions irradiated up to the saturation conditions, topographic changes and a drastic increase of the CL emission are observed (figure 4). CL spectra show that the main mechanism responsible for the luminescence increase depends on the sample considered. As figure 5 shows, the $\mathrm{CL}$ increase in the TH2-850b sample is mainly due to the growth of the $540 \mathrm{~nm}$ emission, while the $430 \mathrm{~nm}$ one shows only a slight intensity increase. In the spectra at $80 \mathrm{~K}$ the different behaviours of both bands are shown. At room temperature, spectra show only the $540 \mathrm{~nm}$ band (figure $5(b)$ ). Figure 6 shows the radiation induced spectral changes in the non-superconducting sample (TKO-930). In contrast to the case shown in figure 5, a moderate increase of both bands is observed. For comparison the spectra of both irradiated samples are shown in figure 7 .

This result agrees with the proposal that the $540 \mathrm{~nm}$ emission is related to oxygen vacancy defects and with the observation in YBCO [9] that irradiation can generate luminescence centres involving pre-existent oxygen vacancies. It has been concluded from EDAX and plasma emission spectroscopy measurements [19,20,22] that the samples used in this work differ in oxygen content as a result of mass losses during the synthesis process. The TH2-850b superconducting sample has a lower oxygen content than the non-superconducting one (TKO-930).

Figures 5-7 show that a lower oxygen content is related to a higher intensity increase of the $540 \mathrm{~nm}$ band during electron irradiation. Such correlation was observed in [9] for YBCO. We suggest that, as in YBCO, electron irradiation generates in $\mathrm{Tl}_{2} \mathrm{Ba}_{2} \mathrm{CuO}_{6+\delta}$ centres involving oxygen vacancies which cause the $540 \mathrm{~nm}$ emission. This band is clearly revealed in spectra recorded at $300 \mathrm{~K}$ as in figure $5(b)$. However, at $80 \mathrm{~K}$ the radiation induced band in the TH2-850b sample (figure 5(a)) appears centred at about $590 \mathrm{~nm}$. This band is complex and includes the $540 \mathrm{~nm}$ band, revealed in figure $5(b)$, and at least one more component at lower energy. The latter would be a consequence of structural changes which take place during the severe irradiation treatment producing changes in the cationic composition of the irradiated area. Due to the 
strong deficiency of thallium and excess of barium detected in this area, the possibility that the material decomposes into $\mathrm{BaCuO}_{2}$ and $\mathrm{BaO}$ phases has been considered.

Barium cuprate $\left(\mathrm{BaCuO}_{2+x}\right)$ has been often detected in sintered 123 HTSC and it was also found after strong laser irradiation of $\mathrm{Hg}$ based superconductors [24]. Comparison of the CL spectrum recorded from the irradiated area in $\mathrm{TH} 2-850 \mathrm{~b}$ with the $\mathrm{CL}$ spectrum of $\mathrm{BaCuO}_{2}$ enables us to rule out the presence of the latter compound in the mentioned area. The $590 \mathrm{~nm}$ luminescence band that appears in the spectrum of the irradiated region (figure $5(a)$ ) is not observed in the spectrum of $\mathrm{BaCuO}_{2}$ (figure 9). In addition, the 500 and $410 \mathrm{~nm}$ CL bands, present in the latter spectrum, were not found in the one recorded in the $\mathrm{Tl}$ based superconductor after electron irradiation. Thus, the $590 \mathrm{~nm}$ band is related to the formation of new phases, different from $\mathrm{BaCuO}_{2}$, or to other irradiation induced structural changes.

In the electron irradiated area an increase of the SEE yield has been observed. This effect was also found in irradiated YBCO [23], and can be explained as a result of the electron irradiation induced changes in the cationic composition and oxygen losses [17]. According to the results summarized in figures 11-13, the increase of the SEE yield could be partially due to the reduction of oxygen content during irradiation. This would agree with the enhancement of the $540 \mathrm{~nm}$ CL band, also related to oxygen content, during irradiation.

In an extended region around the irradiated area, in which cationic composition and topography have not been modified, SEE measurements indicate a decrease of oxygen content. In this region the reduced oxygen content is accompanied by an increase of CL intensity, as compared with CL intensity in unaffected areas. CL images do not show clear changes in the luminescence distribution around the irradiated region, but spectra show that the $540 \mathrm{~nm}$ CL emission band is stronger in the region surrounding the irradiated material than far from it (figure 8). Our observations support the relation between the $540 \mathrm{~nm}$ luminescence band and oxygen depletion, as confirmed by the SEE yield measurements of oxygen deficiency around the irradiated area. These results agree with previous investigations in electron irradiated YBCO ceramics [23]. In [23] thermally driven diffusion processes were proposed as the mechanism leading to the creation of the oxygen depleted area surrounding the irradiated region.

The present observations show that both SEM based techniques, SEE and CL, can be used to study processes related to the oxygen sublattice of TBCO. SEE enables us to detect variations of oxygen content with high spatial resolution while a $\mathrm{CL}$ emission band is related to complex defects involving oxygen vacancies. SEE and CL can provide complementary information on the defect structure in the oxygen sublattice of TBCO. The CL properties of TBCO have been previously discussed [14,21] and compared with similar results concerning other high- $T_{c}$ superconductors. On the other hand SEE results on TBCO samples have not been, to our knowledge, previously reported. For this reason the present results, in particular the qualitative dependence of the yield on oxygen content shown in figure 11, will be discussed in the following.
In order to explain the SEE properties of $\mathrm{Tl}$ based ceramics, the co-existence of two electronic subsystems should be considered: free electrons related to oxygen deficiency (I) and holes (II). The latter are known to be the charge carriers in the $\mathrm{Cu}-\mathrm{O}$ planes. This assumption does not contradict models of electronic processes in HTSCs with oxygen excess, such as BSCCO and TBCO [25-30]. In addition, an interaction between the two subsystems is present when a change in free electron concentration influences the number of holes [28].

We consider the basic formula describing the SEE yield [16]

$$
\Delta=\beta n \lambda B \mathrm{e}^{-\lambda / L}
$$

where $\beta$ is the inelastic scattering cross-section of secondary electrons at primary electrons, $n$ the concentration of free carriers, $\lambda$ the escape depth for secondary electrons (SEs) and $L$ its mean free path. $B$ represents the probability of the SEs overcoming the surface barrier, while $\beta n \lambda$ describes the SE generation. The $\mathrm{e}^{-\lambda / L}$ term describes the SE scattering.

Changes in oxygen content $(x)$ need to be considered as a parameter. The influence of the exponential term is dominant and different mean free paths should be considered for charge carriers which belong to different subsystems (I and II). For the free electrons related to oxygen deficiency $L_{e}=1 / n_{e} \alpha_{e}$, while $L_{h}=1 / n_{h} \alpha_{h}$ for holes. $\alpha_{e}$ and $\alpha_{h}$ represent the inelastic scattering cross-sections of secondary electrons at charge carriers of both subsystems. The dependence of the exponential term $\exp \left[-\lambda\left(\alpha_{e} n_{e}+\alpha_{h} n_{h}\right)\right]$ on $x$ is related to the influence of $x$ on $n_{e}$ and $n_{h}$. When $x$ (and oxygen concentration) decreases, the number of free electrons grows whereas the number of holes drops. These effects would respectively decrease and increase the value of the SEE yield $\Delta$. The net change in $\Delta$ depends on the interaction mechanism between the two subsystems (I and II), initial hole concentration, difference between $\alpha_{e}$ and $\alpha_{h}$ and permissible oxygen losses. For example, in $\mathrm{Bi}_{2} \mathrm{Sr}_{2} \mathrm{CaCu}_{2} \mathrm{O}_{8-x}$ a significant decrease of the hole concentration is observed for small oxygen losses [28], and $\Delta$ increases. Further oxygen losses cause the increase of $n_{e}$, and decrease of $\Delta$. As a consequence, a maximum in the $\Delta(x)$ curve is observed. A qualitative analogy has been found in our Tl based ceramics (figure 11). The model described here is valid if the electronic processes related to oxygen losses in this material lead to a decrease of the hole concentration and to an increase of the number of free electrons. The first seems to be experimentally proved, (see e.g. $[25,27,29,30])$. The existence of free electrons after oxygen loss seems to be natural independent of the origin of such a deficiency (vacancy generation, removal of interstitial oxygen atoms, etc). These considerations have preliminary character, and further theoretical and experimental studies should be performed to explain in detail the complex behaviour of the $\Delta$ function.

\section{Conclusions}

Electron irradiation of $\mathrm{Tl}_{2} \mathrm{Ba}_{2} \mathrm{CuO}_{6+\delta}$ (TBCO) in the SEM causes changes in the cationic stoichiometry as well as variations in oxygen content. Irradiated areas have been found 
to be $\mathrm{Tl}$ poor and show a relative increase of $\mathrm{Ba}$ content. CL spectroscopy shows that irradiation causes the increase of a $540 \mathrm{~nm}$ emission band attributed to complex defects involving oxygen vacancies. Other features of the CL spectra suggest the presence of radiation induced new phases.

The value of the SEE yield, $\Delta$, has been found to depend on the oxygen content of the samples. The dependence can be explained on the basis of two electron subsystems and their mutual interaction. Measurements of $\Delta$ enable us to study variations of oxygen content in the samples with high spatial resolution. In the case of irradiated samples it was observed that variations of the oxygen content extend in some cases up to a distance of about $1000 \mu \mathrm{m}$ from the electron irradiated area. This effect should be taken into account in the development of lithographic and paterning processes in high- $T_{c}$ superconductors.

In the oxygen deficient region that surrounds the irradiated material, an enhancement of the oxygen deficiency related CL band has been observed. This supports the relation between this $540 \mathrm{~nm}$ luminescence band and oxygen depletion, as confirmed by the SEE yield measurements.

\section{Acknowledgments}

The authors thank S G Prutchenko for EDX measurements. CDG thanks the Spanish MEC for an FPI research grant. This work has been supported by DGICYT (project PB 93-1256), CICYT (project MAT 95-1184-E), NATO (grant HTECH. LG 941034), the Russian Foundation of Fundamental Research (project 94-03-0802) and the Russian State Programme on High $T_{c}$ Superconductivity (project 93079).

\section{References}

[1] Tolpygo S, Nadgorny B, Shokhor S, Tafuri F, Lin Y, Bourdillon A and Gurvitch M 1993 Physica C 209211

[2] Auciello O, Kraus A R, Santiago Avilés J, Schreiner A F and Gruen D M 1988 Appl. Phys. Lett. 52239

[3] Matsui S, Ichihashi T, Yoshitake T, Miura S, Satoh T and Mito M 1990 J. Vac. Sci. Technol. B 81771

[4] Shen Y Q, Freltoft T and Vase P 1991 Appl. Phys. Lett. 59 1365
[5] Sobolewski R, Xiong W, Kula W and Gavaler J R 1994 Appl. Phys. Lett. 64643

[6] Kawamoto K and Hirabayashi I 1994 Phys. Rev. B 493655

[7] Osquiguil E, Maenhoudt M, Wuyts B, Bruynseraede Y, Lederman D and Schuller I K 1994 Phys. Rev. B 49 3675

[8] Gómez P, Jiménez J, Martín P, Piqueras J and Domínguez-Adame F 1993 J. Appl. Phys. 746289

[9] Gómez P, Piqueras J, Sayagués M J and González-Calbet J M 1995 Solid State Commun. 9645

[10] Torres A, Jiménez J, Gómez P and Piqueras J 1995 Mat. Res. Soc. Symp. Proc. vol 373 (Pittsburgh, PA: Materials Research Society) p 431

[11] Cooke D W, Rempp H, Fisk Z, Smith J L and Jahan M S 1987 Phys. Rev. B 362287

[12] Stankevitch V G et al 1993 Phys. Rev. B 471024

[13] Fugol I, Politis C, Ratner A, Samovarov V and Zhuravlev V 1994 J. Lumin. 62291

[14] Gómez P, Piqueras J and Opagiste C 1994 Solid State Commun. 91747

[15] Tomashpolsky Yu Ya, Sevostyanov M A, Sadovskaya N V and Kolganova N V 1989 J. Microsc. Spectrosc. Electron. 14213

[16] Tomashpolsky Yu Ya 1993 Ferroelectrics 144231

[17] Tomashpolsky Yu Ya and Sadovskaya N V 1995 Ferroelectrics 163129

[18] Tomashpolsky Yu Ya and Sadovskaya N V 1995 Ferroelectrics 17097

[19] Opagiste C, Couach M, Khoder A F, Abraham R, Jondo T K, Jorda J L, Cohen-Adad M Th, Junod A, Triscone G and Müller J 1993 J. Alloys Compounds. 19547

[20] Opagiste C, Triscone G, Couach M, Jondo T K, Jorda J L, Junod A, Khoder A F and Müller J 1993 Physica C 213 17

[21] Díaz-Guerra C, Piqueras J and Opagiste C 1996 Physica C 259121

[22] Opagiste C et al 1993 Physica C 205247

[23] Díaz-Guerra C, Piqueras J, Tomashpolsky Yu Ya and Sadovskaya N V 1996 Phys. Status Solidi a 155525

[24] Ren Y Y, Chang H, Xiong Q, Xue Y Y and Chu C W 1994 Physica C 226209

[25] Shimakawa Y, Kubo Y, Manako T and Igarashi H 1989 Phys. Rev. B 4011400

[26] Martin C, Maignan A, Provost J, Michel C, Gerveu M, Tournier B and Braveau B 1990 Physica C 1688

[27] Shimakawa Y, Kubo Y, Manako T, Igarashi H, Izumi F and Asano H 1990 Phys. Rev. B 4210165

[28] Nagoshi M, Suzuki T, Fukuda Y, Terashima K, Nakanishi Y, Ogita M, Tokiwa A, Syono Y and Tachiki M 1991 Phys. Rev. B 4310445

[29] Kubo Y, Shimakawa Y, Manako T and Igarashi H 1991 Phys. Rev. B 437875

[30] Shimakawa Y 1993 Physica C 204247 